\title{
HDL particles from type 1 diabetic patients are unable to reverse the inhibitory effect of oxidised LDL on endothelium-dependent vasorelaxation
}

\author{
L. Perségol • M. Foissac $\cdot$ L. Lagrost • A. Athias • \\ P. Gambert $\cdot$ B. Vergès $\cdot$ L. Duvillard
}

Received: 27 March 2007 / Accepted: 25 July 2007 / Published online: 12 September 2007

(C) Springer-Verlag 2007

\begin{abstract}
Aims/hypothesis In healthy individuals, HDL can counteract the inhibition of vasorelaxation induced by oxidised LDL. Several abnormalities such as increased size, glycation and decreased paraoxonase activity have been reported for HDL from type 1 diabetic patients. Thus, we hypothesised that the ability of HDL to protect vessels against impairments of vasorelaxation would be decreased in these patients.

Methods We compared the ability of HDL from 18 type 1 diabetic patients and 12 control participants to counteract the inhibition of endothelium-dependent relaxation induced by oxidised LDL on rabbit aorta rings.

Results Serum triacylglycerol and total cholesterol, LDLand HDL-cholesterol were similar in type 1 diabetic and control participants. Fasting glycaemia and the HDLfructosamine level were higher in diabetic patients than in
\end{abstract}

L. Perségol and M. Foissac contributed equally to this study.

L. Perségol • M. Foissac · L. Lagrost • P. Gambert • B. Vergès •

L. Duvillard

INSERM Research Center 866,

Dijon, France

L. Perségol $\cdot$ M. Foissac $\cdot$ L. Lagrost $\cdot$ A. Athias $\cdot$ P. Gambert

B. Vergès $\cdot$ L. Duvillard

University of Burgundy, IFR 100,

Dijon, France

B. Vergès

Department of Endocrinology and Metabolic Diseases,

CHU Dijon Bocage Hospital,

Dijon, France

L. Duvillard $(\bowtie)$

Department of Medical Biochemistry,

CHU Dijon Bocage Hospital,

P.O. Box 77908, 21079 Dijon Cedex, France

e-mail: laurence.duvillard@chu-dijon.fr controls $(9.06 \pm 3.55$ vs $5.27 \pm 0.23 \mathrm{mmol} / \mathrm{l}, p<0.005$; and $10.2 \pm 3.2 \mathrm{vs} 7.7 \pm 2.5 \mu \mathrm{mol} / \mathrm{g}$ protein, $p<0.05$, respectively). HDL composition, size and paraoxonase activity were similar in both groups. HDL from controls reduced the inhibitory effect of oxidised LDL on maximal relaxation $\left(E_{\max } ; 79.3 \pm\right.$ 11.8 vs $66.4 \pm 11.7 \%, p<0.05)$, whereas HDL from type 1 diabetic patients had no effect $\left(E_{\max }=70.6 \pm 17.4\right.$ vs $63.9 \pm$ $17.2 \%$, NS). In type 1 diabetic patients, $E_{\max }$ was not correlated with glycaemia or the HDL-fructosamine level. Conclusions/interpretation HDL particles from type 1 diabetic patients do not protect against inhibition of endothelium-dependent vasorelaxation induced by oxidised LDL, in contrast to HDL particles from healthy individuals. This defect cannot be explained by abnormalities in HDL composition, size or paraoxonase activity, and may contribute to the early development of atherosclerotic lesions in type 1 diabetic patients.

Keywords Aorta rings · Endothelium-dependent vasorelaxation $\cdot \mathrm{HDL} \cdot$ Oxidised LDL $\cdot$ Rabbit · Type 1 diabetes mellitus

$\begin{array}{ll}\text { Abbreviations } \\ E_{\max } & \text { maximal relaxation } \\ \text { NO } & \text { nitric oxide } \\ \text { ox-LDL } & \text { oxidised LDL } \\ \text { PON } & \text { paraoxonase }\end{array}$

\section{Introduction}

An increased cardiovascular risk has been demonstrated in type 1 diabetic patients. However, the mechanisms likely to explain such a higher risk are far from being totally elucidated [1]. 
Decreased endothelium-dependent vasorelaxation, an abnormality often observed at an early stage of the development of atherosclerosis, has been described in these patients [2].

Some papers have reported quantitative and qualitative abnormalities in type 1 diabetic patients treated by subcutaneous insulin. With regard to HDL, HDL-cholesterol has been demonstrated to be moderately increased and HDL particles to be larger than in healthy individuals with a higher $\mathrm{HDL}_{2}: \mathrm{HDL}_{3}$-cholesterol ratio $[3,4]$. Moreover, glycation of HDL is likely to be increased in type 1 diabetic patients, owing to hyperglycaemia. Finally, a decrease in paraoxonase (PON) activity has also been reported [5].

Due to these multiple modifications, HDL particles from type 1 diabetic patients may be less protective against impairments of vasorelaxation than HDL particles from healthy individuals [6]. In the present study we investigated this possibility using isolated aorta rings, a previously validated model.

\section{Methods}

Participants Eighteen type 1 diabetic patients and 12 normolipidaemic and normoglycaemic controls were included in this protocol, which was approved by the Ethics Committee of Dijon University Hospital. Written informed consent was obtained from each participant before the study. With the exception of insulin for type 1 diabetic patients, none of the participants were taking any antioxidants or any drugs that could affect lipid metabolism. All participants were non-smokers. Diabetes duration was $15.5 \pm 9.7$ years. Among type 1 diabetic patients, four were treated by insulin pump and 14 by insulin injections four times a day. Four type 1 diabetic patients had retinopathy and two had neuropathy. Two patients had microalbuminuria in the range of 20-25 mg/l; four had high blood pressure. Controls were included in our study if they had a fasting glycaemia $<6.10 \mathrm{mmol} / 1$, serum triacylglycerol $<1.7 \mathrm{mmol} / 1$ and HDL-cholesterol $>1.04$ for men and $>1.30 \mathrm{mmol} / 1$ for women.

Biochemical measurements Glycaemia, $\mathrm{HbA}_{1 \mathrm{c}}$, lipid parameters and PON were measured as previously described [6]. Apolipoprotein E and apolipoprotein CIII were measured using Immuno Chimie International reagents (Bouffémont, France) on a Dimension analyser (Dade Behring, Newark, DE, USA). Fructosamine in the HDL was quantified using ABX Diagnostics reagents (Montpellier, France) on a DU 64 spectrophotometer (Beckman Coulter, Palo Alto, CA, USA). The sphingomyelin:lecithin ratio in the HDL was measured by liquid chromatography/mass spectrometry as previously described [7].
Size distribution of $H D L$ The relative proportions of the different HDL subfractions $\left(\mathrm{HDL}_{2 \mathrm{~b}}\right.$ 9.71-12.90 nm; $\mathrm{HDL}_{2 \mathrm{a}}$ 8.77-9.71 nm; HDL 3 a 8.17-8.77 nm; HDL $3 \mathrm{~b}$ 7.76$8.17 \mathrm{~nm}$; and $\mathrm{HDL}_{3 \mathrm{c}} 7.21-7.76 \mathrm{~nm}$ ) were determined by polyacrylamide gradient gel electrophoresis as previously described [8].

Preparation of lipoproteins The different lipoprotein subclasses were isolated from serum or plasma by sequential flotation ultracentrifugation, according to their density [6]. The oxidation of LDL was performed by incubating freshly prepared LDL from normolipidaemic donors as previously described [6].

Table 1 Clinical and biochemical characteristics, HDL composition and size

\begin{tabular}{|c|c|c|}
\hline & $\begin{array}{l}\text { Controls } \\
(n=12)\end{array}$ & $\begin{array}{l}\text { Type } 1 \\
\text { diabetic patients } \\
(n=18)\end{array}$ \\
\hline \multicolumn{3}{|l|}{ Clinical characteristics } \\
\hline Age (years) & $35 \pm 12$ & $35 \pm 14$ \\
\hline Sex (male/female) & $7 / 5$ & $10 / 8$ \\
\hline BMI $\left(\mathrm{kg} / \mathrm{m}^{2}\right)$ & $23.6 \pm 1.4$ & $24.0 \pm 3.0$ \\
\hline Fasting glycaemia (mmol/l) & $5.27 \pm 0.23$ & $9.06 \pm 3.55 * *$ \\
\hline $\mathrm{HbA}_{1 \mathrm{c}}(\%)$ & ND & $8.47 \pm 1.34$ \\
\hline Total cholesterol (mmol/l) & $5.43 \pm 0.57$ & $5.02 \pm 0.93$ \\
\hline HDL-cholesterol (mmol/l) & $1.49 \pm 0.40$ & $1.49 \pm 0.37$ \\
\hline LDL-cholesterol (mmol/l) & $3.60 \pm 0.50$ & $3.13 \pm 0.86$ \\
\hline Triacylglycerol (mmol/l) & $0.80 \pm 0.41$ & $0.69 \pm 0.30$ \\
\hline Apolipoprotein AI (g/l) & $1.57 \pm 0.25$ & $1.55 \pm 0.38$ \\
\hline Apolipoprotein B (g/l) & $0.92 \pm 0.19$ & $0.85 \pm 0.18$ \\
\hline \multicolumn{3}{|l|}{ HDL } \\
\hline Free cholesterol (\%) & $3.4 \pm 0.7$ & $4.1 \pm 0.9$ \\
\hline Cholesteryl ester (\%) & $24.1 \pm 1.1$ & $23.3 \pm 2.0$ \\
\hline Triacylglycerol (\%) & $2.6 \pm 1.2$ & $2.6 \pm 1.5$ \\
\hline Protein $(\%)$ & $44.5 \pm 4.4$ & $46.0 \pm 4.8$ \\
\hline Apolipoprotein AI (\%) & $35.9 \pm 3.7$ & $36.1 \pm 3.9$ \\
\hline Apolipoprotein CIII (\%) & $1.94 \pm 0.29$ & $2.29 \pm 0.29$ \\
\hline Apolipoprotein E (\%) & $0.27 \pm 0.08$ & $0.28 \pm 0.09$ \\
\hline Phospholipid (\%) & $25.6 \pm 4.5$ & $24.0 \pm 5.3$ \\
\hline Sphingomyelin:lecithin & $0.15 \pm 0.05$ & $0.12 \pm 0.03$ \\
\hline Fructosamine $(\mu \mathrm{mol} / \mathrm{g}$ protein $)$ & $7.7 \pm 2.5$ & $10.2 \pm 3.2 *$ \\
\hline $\begin{array}{l}\text { PON activity (nmol min } \\
{\left[\mathrm{mg} \text { Apolipoprotein } \mathrm{AI}^{-1}\right] \text { ) }}\end{array}$ & $1.3 \pm 1.0$ & $1.6 \pm 1.1$ \\
\hline $\mathrm{HDL}_{2 \mathrm{~b}}(\%)$ & $25.0 \pm 9.1$ & $22.5 \pm 8.7$ \\
\hline $\mathrm{HDL}_{2 \mathrm{a}}(\%$ & $28.9 \pm 2.6$ & $27.1 \pm 4.1$ \\
\hline $\mathrm{HDL}_{3 \mathrm{a}}(\%)$ & $26.6 \pm 5.0$ & $26.0 \pm 3.3$ \\
\hline $\mathrm{HDL}_{3 \mathrm{~b}}(\%)$ & $13.4 \pm 3.9$ & $15.1 \pm 5.3$ \\
\hline $\mathrm{HDL}_{3 \mathrm{c}}(\%)$ & $6.2 \pm 2.8$ & $9.2 \pm 5.9$ \\
\hline
\end{tabular}

Values are expressed as mean $\pm \mathrm{SD}$; HDL composition is expressed as the percentage of total HDL mass.

Apo Apolipoprotein; $N D$ not determined

$* p<0.05$

$* * p<0.005$ 
Vasoreactivity on rabbit aorta rings Vasoreactivity experiments were performed on rabbit (Charles River, L'Arbresle, France) aorta rings following a previously described protocol [6]. This experiment has been approved by the local Ethics Committee for animals at Dijon University.

Statistical analysis Statistical analysis was performed using Statview statistical software (SAS Institute, Berkeley, CA, USA). Data are expressed as means \pm SD. The comparison of data from type 1 diabetic patients and control participants was performed by the Mann-Whitney $U$ test. The maximal relaxation $\left(E_{\max }\right)$ values obtained in two different conditions and the same experiment were compared by the non-parametric Wilcoxon matched-pair test. Correlation coefficients were performed by linear regression analysis.

\section{Results}

Clinical and biochemical characteristics As seen in Table 1, the type 1 diabetic patients had a broad range of glycaemic control, with fasting glycaemia ranging from 4.98 to $17.82 \mathrm{mmol} / 1$ and $\mathrm{HbA}_{1 \mathrm{c}}$ from 6.9 to $11.7 \%$. Lipid parameters did not differ between type 1 diabetic patients and control participants.

HDL composition Table 1 shows that the composition, size and PON activity of HDL was identical in the two groups of participants. In contrast, the HDL-fructosamine level was $32 \%$ higher in type 1 diabetic patients than in controls $(10.2 \pm$ 3.2 vs $7.7 \pm 2.5 \mu \mathrm{mol} / \mathrm{g}$ protein, $p<0.05$ ).

Ability of $H D L$ to reverse the ox- $L D L$-induced inhibition of endothelium-dependent vasodilation As seen in Fig. 1, no significant difference was observed for segments pre-

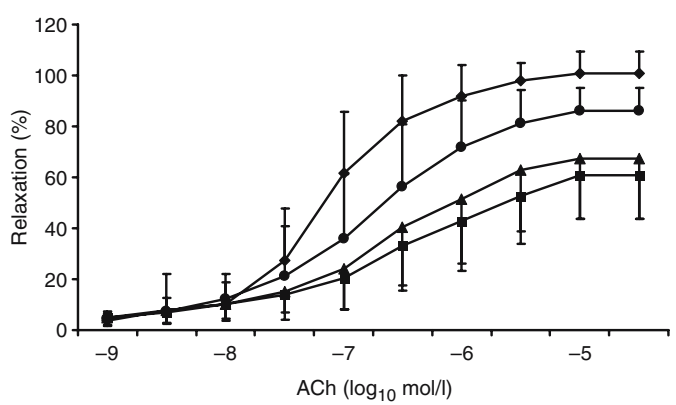

Fig. 1 Influence of HDL from type 1 diabetic patients and controls on the inhibitory effect of ox-LDL on endothelium-dependent vasorelaxation in rabbit aorta rings. Mean concentration-response curves for acetylcholine $(\mathrm{ACh})$ in aortas contracted with $0.3 \mu \mathrm{mol} / 1$ arterenol hydrochloride and pre-incubated for $2 \mathrm{~h}$ either with ox-LDL alone ( $1 \mathrm{~g}$ protein/l; squares) or with ox-LDL and HDL from controls (circles) or with ox-LDL and HDL from type 1 diabetic patients $(1 \mathrm{~g}$ protein/l; triangles) or in Krebs buffer (diamonds). Values represent means $\pm \mathrm{SD}$ incubated with HDL from controls or type 1 diabetic patients when compared with the $E_{\max }$ obtained in segments preincubated in Krebs buffer $\left(E_{\max }=100.6 \pm 1.0\right.$ vs $96.3 \pm 4.3 \%$ and $100.9 \pm 3.4$ vs $93.5 \pm 5.8 \%$ for control and type 1 diabetic persons, respectively; data not shown). The $E_{\max }$ was greater after preincubation with HDL from controls + ox-LDL than after preincubation with ox-LDL alone $\left(E_{\max }=\right.$ $79.3 \pm 11.8$ vs $66.4 \pm 11.7 \%, p<0.01)$. In contrast, the addition of HDL from type 1 diabetic patients to ox-LDL did not modify the $E_{\max }$, compared with incubation with ox-LDL $\left(E_{\max }=70.6 \pm 17.4\right.$ vs $63.9 \pm 17.2 \%$, NS).

Correlations In type 1 diabetic patients, the $E_{\max }$ obtained after co-incubation of the vessels with ox-LDL and HDL was correlated neither with fasting glycaemia ( $r=0.29, \mathrm{NS})$, nor with $\mathrm{HbA}_{1 \mathrm{c}}(r=0.02$, NS), nor with the HDL-fructosamine level $(r=0.23$, NS).

\section{Discussion}

Although plasma HDL-cholesterol is normal or slightly elevated in type 1 diabetic patients, the early development of atherosclerosis could be due to qualitative or functional abnormalities of HDL particles. In the present study, we report for the first time a functional defect of HDL from type 1 diabetic patients. This defect consists of loss of the ability to protect arteries from ox-LDL-induced inhibition of endothelium-dependent relaxation.

The inhibitory effect of ox-LDL and of some of their specific compounds, such as derivatives of cholesterol oxidised in position 7, on endothelium-dependent vasorelaxation is mainly related to decreased bioavailability of nitric oxide (NO) [9].

HDL particles are likely to counteract the deleterious effect of ox-LDL on vascular relaxation both by inhibiting the LDL oxidation process and stimulating NO production. Indeed, lipid peroxides and oxidised derivatives of cholesterol are transferred from LDL to HDL, where they can be hydrolysed by HDL-associated enzymes such as PON and platelet-associated-factor acetyl hydrolase. As far as NO production is concerned, HDL particles are capable of stimulating endothelial NO synthase, after binding to cell surface receptors such as scavenger receptor $\mathrm{BI}$ or the lysophospholipid receptor $\mathrm{S}_{3} \mathrm{P}_{3}$ [6].

The reasons for the loss of the vasorelaxant effect of HDL particles in type 1 diabetic patients are not clear, but our work allows us to exclude some of the possible causes. Some papers, but not all, have reported an increase in the $\mathrm{HDL}_{2}$-:HDL $\mathrm{H}_{3}$-cholesterol ratio in type 1 diabetic patients $[3,4,10]$. As $\mathrm{HDL}_{2}$ particles have been demonstrated to have a lower antioxidant capacity than $\mathrm{HDL}_{3}$, a shift of the 
HDL profile toward $\mathrm{HDL}_{2}$ particles could have induced a decrease in the vasorelaxant effect of HDL [11]. Modifications of HDL composition are also likely to impair the ability of HDL to counteract the inhibition of vascular relaxation by ox-LDL, as suggested by our recent work in type 2 diabetic patients, in whom the vasorelaxant effect was lost with a strong negative correlation between HDL triacylglycerol enrichment and HDL vasorelaxant ability [6]. However, we have demonstrated here that the decreased ability of HDL to counteract the ox-LDL-induced inhibition of vascular relaxation in type 1 diabetic patients cannot be explained by a change in HDL size or composition.

Moreover, in our type 1 diabetic patients PON activity was not decreased and cannot therefore be involved in the loss of the vasorelaxant effect we observed.

Glycation, consisting of the covalent binding of glucose to free amine groups of proteins, is likely to induce conformational modifications of HDL that could impair their binding to the cell surface receptors involved in the signalling pathways that activate endothelial NO synthase. In this work we observed a $32 \%$ increase in the HDLfructosamine level in type 1 diabetic patients compared with controls. However, we did not observe any negative correlation between $E_{\max }$ and the HDL-fructosamine level in type 1 diabetic patients. Thus, structural changes induced by glycation are unlikely to explain the decreased vasorelaxant effect of HDL observed by us in these patients.

Besides, compared with HDL particles from control participants, HDL particles from type 1 diabetic patients have been demonstrated to be less effective in removing lipoperoxides from red blood cells $[5,12]$. This abnormality appears to be independent of changes in PON activity, but related to glyco-oxidation [12]. Thus, a decreased ability of HDL particles to remove lipoperoxides from ox-LDL could at least partly explain the lesser vasorelaxant effect of HDL particles from type 1 diabetic patients.

In conclusion, the present study demonstrates that the ability of HDL particles from type 1 diabetic patients to counteract the inhibitory effect of ox-LDL on endotheliumdependent vasorelaxation is impaired. This may contribute to the early development of atherosclerotic lesions, which are more frequently observed in these patients than in the healthy population.

Acknowledgements The authors wish to thank D. Battault, E. Niot and L. Princep for their expert technical assistance. This study was supported by the Institut National de la Santé et de la Recherche Médicale (INSERM; the National Institute of Health and Medical Research), the University of Bourgogne and the Regional Council of Bourgogne.

Duality of interest The authors declare that there is no duality of interest associated with this manuscript.

\section{References}

1. Laing SP, Swerdlow AJ, Slater SD et al (2003) Mortality from heart disease in a cohort of 23,000 patients with insulin-treated diabetes. Diabetologia 46:760-765

2. Clarkson P, Celermajer DS, Donald AE et al (1996) Impaired vascular reactivity in insulin-dependent diabetes mellitus is related to disease duration and low density lipoprotein cholesterol levels. J Am Coll Cardiol 28:573-579

3. Colhoun HM, Otvos JD, Rubens MB, Taskinen MR, Underwood SR, Fuller JH (2002) Lipoprotein subclasses and particle sizes and their relationship with coronary artery calcification in men and women with and without type 1 diabetes. Diabetes 51:1949-1956

4. Dunn FL (1992) Plasma lipid and lipoprotein disorders in IDDM. Diabetes 41(Suppl 2):102-106

5. Ferretti G, Bacchetti T, Busni D, Rabini RA, Curatola G (2004) Protective effect of paraoxonase activity in high-density lipoproteins against erythrocyte membranes peroxidation: a comparison between healthy subjects and type 1 diabetic patients. J Clin Endocrinol Metab 89:2957-2962

6. Perségol L, Vergès B, Foissac M, Gambert P, Duvillard L (2006) Inability of HDL from type 2 diabetic patients in counteracting the inhibitory effect of oxidized LDL on endothelium-dependent vasorelaxation. Diabetologia 49:1380-1386

7. Delmas D, Rebe C, Lacour S et al (2003) Resveratrol-induced apoptosis is associated with Fas redistribution in the rafts and the formation of a death-inducing signaling complex in colon cancer cells. J Biol Chem 278:41482-41490

8. Lagrost L, Athias A, Herbeth B et al (1996) Opposite effects of cholesteryl ester transfer protein and phospholipid transfer protein on the size distribution of plasma high density lipoproteins. Physiological relevance in alcoholic patients. J Biol Chem 271:19058-19065

9. Deckert V, Brunet A, Lantoine F et al (1998) Inhibition by cholesterol oxides of NO release from human vascular endothelial cells. Arterioscler Thromb Vasc Biol 18:1054-1060

10. Winocour PH, Durrington PN, Ishola M, Anderson DC (1986) Lipoprotein abnormalities in insulin-dependent diabetes mellitus. Lancet 24;1:1176-1178

11. Kontush A, Chantepie S, Chapman MJ (2003) Small, dense HDL particles exert potent protection of atherogenic LDL against oxidative stress. Arterioscler Thromb Vasc Biol 23:1881-1888

12. Kalogerakis G, Baker AM, Christov S et al (2005) Oxidative stress and high-density lipoprotein function in type I diabetes and end-stage renal disease. Clin Sci 108:497-506 